\title{
AGENDA DES CONGRES
}

18-19 mars 1994

13th International Symposium of Operative Andrology : Traditional and Microsurgical Interventions in Andrology.

Budapest, Hongrie.

Contact : Congress Bureau Motesz, P.O. Box 145, Budapest.

Tél : 361-251-7999, Fax : 361-183-7918.

\section{1-22 mars 1994}

Premier Congrès National de la Société Italienne d'Andrologie Médicale.

Modène, Italie.

Contact : Prof. Paolo Marama, Cattedra di Endocrinologia, Clinica Medica I, Universita di Modena, Via del pozzo 71, 41100, Modena.

Tél : 39-59-379-928, Fax : 39-59-363-114.

\section{5-28 mars 1994}

\section{American Society of Andrology $29^{\text {th }}$ Annual Meeting}

Springfield, Illinois, U.S.A.

Contact : Dr. Armando Amador, Department of Obstetrics/Gynecology, SIV School of Medicine, P.O. Box 19230, Springfield, Illinois.

Tél : 217-782-9306, Fax : 217-782-8246.

\section{Avril 1994}

Séance commune de la Société Française de Gynécologie et de la SALF sur le thème : LE SYNDROME DE KLINEFELTER

Salle Lavoisier - Faculté de Médecine

3 ème étage, 45, rue des Saints Pères

Paris VI

Coordinateurs : Ch. Sultan et J.Belaisch.

\section{1-22 Avril 1994}

International Symposium (Ares Serono) on : Male factor in infertility.

Hôpital américain de Neuilly sur Seine.

Contact : FA.SI. Congress, Viale Gorizia 24. 00198 Rome.

Tél : 39 (6) 854-0296/8417001, Fax : 39 (6) 841-4495.

\section{5-7 mai 1994}

7th International Urological Symposium : Andrology Symposium in Memory of Prof. Dr. Alpay Kelâmi.

North Cyprus, Turquie.

Contact : Dr Kemal Bolayir, P.O. Box 597, Lefkasa-Kibris, Mersin 10. 
VI World Meeting on Impotence : Trends and Innovations in Pathophysiology and Management of Impotence.

Singapour.

Contact : The Secretariat (6th WMI), Obstetrical and Gynaecological, Society of Singapore, c/o Department of Obstetrics and Gynaecology, National University Hospital, Lower Kent Ridge Road, Singapore 0511, République de Singapour.

Tél : 65-777-0313, Fax 65-779-4753.

\section{9-24 Septembre 1994}

VIth National Congress of the Indonesian Society of Andrology and III International Symposium on New Prospectives of Andrology in Human Reproduction.

Mando, North Sulawesi, Indonésie.

Contact : Secrétariat, lab./UPF Internal Medicine, School of Medicine Sam Ratulangi University, Academic Hospital “Gunung Menang“, J1, Surdirman Manado.

\section{8-14 Octobre 1994}

7th International Symposium on Spermatology.

Cairns, Australie.

Contact : Australian Convention and Travel Services, G.P.O., Box 2200, AusCanberra A.C.T. 2601.

\section{1-3 Décembre 1994}

Congrès de la Société d'Andrologie de Langue Française.

Deauville.

Contact : Pr. M. Drosdowsky, Laboratoire de Biochimie, Faculté de Médecine, 14032 Caen, cedex.

Tél : 31-06-45-53, Fax 31-44-78-26. 